\title{
Study on the Chemical Constituents and anti-HBV Activity of Hypericum japonica
}

\author{
Gao-yun Chen ${ }^{1}$, Min Liu ${ }^{1}$, Meng-bin Yu ${ }^{1}$, Chao Zheng ${ }^{2}$ and Si-chen Wei ${ }^{3}$ \\ 1 Institute of Chemical Defense, Beijing, PR China \\ 2 Department of Biology, Shantou University, Shantou, PR China \\ ${ }^{3}$ Faculty of Science of Beijing University of Chemical Technonlogy, Beijing ,PR China
}

\begin{abstract}
The most common cause of chronic viral hepatitis worldwide is from the Hepatitis B virus (HBV). Screening the safe and effective anti-HBV compounds in traditional Chinese medicine provides a large opportunity for the development of anti-HBV drugs. We found the ethanol extract of $H$. japonica had the besr inhibitory effects on $\mathrm{HBeAg}$ secretion from 20 species of medicinal plants In this study, silica gelSephadex LH-20. and a thin layer of chromatography were used to screen and isolate active anti-hepatitis B virus compounds from Hypericum japonica that was extracted with $60 \%$ ethanol. Four compounds were separated from the ethyl acetate extract of $H \bullet$ japonica and their structures were identified by spectral chemical and structural analyses. In addition, using HepG2 2.2.15 cell line with the cytotoxicity and inhibitory effecting on the secretion of $\mathrm{HBeAg}$ and $\mathrm{HBsAg}$ of the 4 isolated compounds were examined by MTT and enzyme-linked immunosorbent assays. The four isolated compounds were determined and 1,3,6,7-tetrahydroxy-9H-xanthen-9one (Compound 4) exhibited the highest activity in inhibiting secretion of HBeAg in HepG2 2.2.15cells. The results indicated a $67.66 \%$ of inhibition rate, $40.25 \mu \mathrm{g} / \mathrm{mL}$ of IC50 value and TI $>2$. We conducted a qualitatively evaluated research using duck hepatitis B virus model in vivo, the results indicated that ethanol extracts of $H$. japonica had anti-HBV effects and almost no sign of disease rebound after drug withdrawing.But the compound 4 has no direct inhibitory effect on HBV-DNA in duck experiment.
\end{abstract}

\section{Introduction}

The Hepatitis B virus (HBV) was discovered in 1966, but even after 50 years, it is still a major global public health problem[1], especially in Asian and African countries. Approximately 350-400 million people are affected with HBV in the world, and up to 1 million suffer from are HBV-related complications every year, including viral hepatitis and hepatocellular carcinoma(HCC)[2]. According to a national serological survey in 2006, China has one of the highest carrier rates of the Hepatitis B surface antigen ( $\mathrm{HBsAg}$ ), reaching $7.18 \%$ of the general population [2-7]. Currently, a great progress is being made in the treatment of the HBV with the development of effective and well-tolerated nucleotide analogues (NAs). However, the treatments of $\mathrm{HBV}$, relying on some limited available antiviral drugs, are not successful in most of the cases. There are still some issues with the progress. First, there are still no highly effective anti-HBV drugs to remove the HBVDNA from chronic infections completely. Second, the clinical anti-HBV drugs have some side effects.

However, anti-HBV drugs of constituents from traditional Chinese herbs have some unique advantages such as high efficiency, low toxicity, and multi-target points. Therefore, screening safe and effective anti-HBV constituents in herbs is an important and necessary aspect within the research of anti-HBV drugs.

We screened out four herbs within this study: (Hypericum japonica, Fagopyrum dibotrys (D.Don) Hara, Areca catechu L and Parmelia saxatile (L.) Ach) with anti-HBV activity from twenty kinds herbs. The result indicated that Hypericum japonica has the potential to suppress the secretion of $\mathrm{HBeAg}$ effectively in a dose-dependent method. In addition, its inhibition rate was the highest out of the four herbs that were screened.

\section{Materials and Methods}

In this Duck-Model, Beijing ducks were infected with duck hepatitis B virus (DHBV) used to evaluate antiHBV activity in this study. HepG2 2.2.15 cell line was used in this study to screen the ethanol extracts from these plants using in vitro assays. The cytotoxicity was examined by MTT assay, and the effects on the secretion of $\mathrm{HBeAg}$ and $\mathrm{HBsAg}$ was examined by enzyme-linked immunosorbent assay. 


\section{Results}

\subsection{Screening of the herbs with anti-HBV activity}

Within this study, the "Records of Li Folk Medicine," was used to identify 20 species of medicinal plants (table 1) and were selected to screen the herds with anti-HBV activity in our study. HepG2 2.2.15 cell line was used to screen the ethanol extracts from these plants using in vitro assays[8-11]. The cytotoxicity was examined by MTT assay, and the effects on the secretion of $\mathrm{HBeAg}$ and HBsAg were examined by enzyme-linked immunosorbent assay (ELISA).

The data indicated that H.japonica could suppress the secretion of $\mathrm{HBeAg}$ effectively in the dose-dependent method and its inhibition rate was $67.66 \%$, the IC50 value was $40.25 \mu \mathrm{g} / \mathrm{mL}$.

Table 1. Inhibitory effects of 20 species of herbs on HBsAg and HBeAg secretion in highest non-toxic concentration in HepG2

2.2 .15 cells $(\bar{x} \pm s, \mathrm{n}=3)$

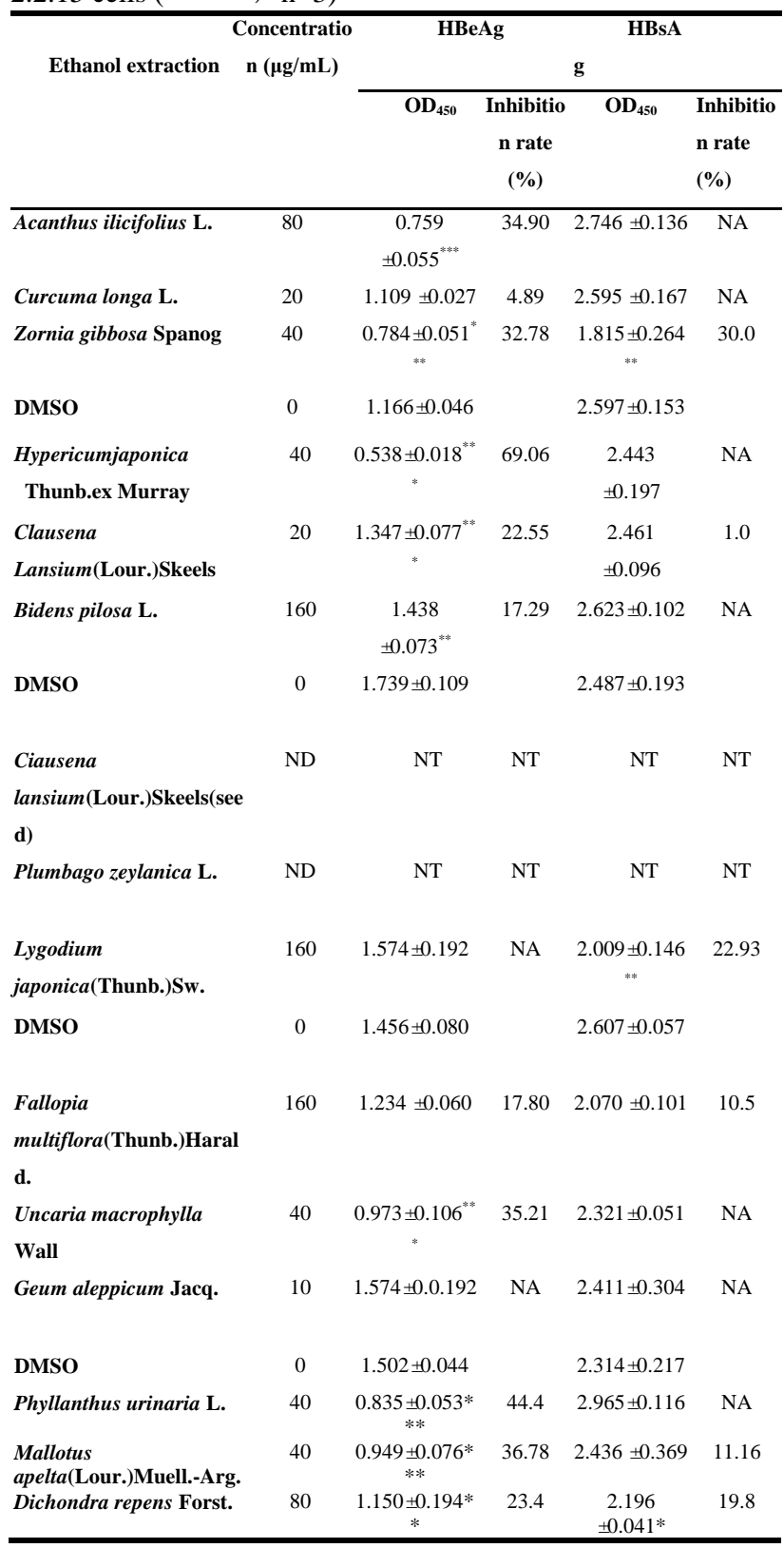

\begin{tabular}{|c|c|c|c|c|c|}
\hline DMSO & 0 & $1.502 \pm 0.044$ & & $2.742 \pm 0.170$ & NA \\
\hline $\begin{array}{l}\text { Emilia } \\
\text { sonchifolia }(\text { L.)DC. }\end{array}$ & 80 & $\begin{array}{c}1.279 \pm 0.035^{*} \\
*\end{array}$ & 20.96 & $2.769 \pm 0.119$ & NA \\
\hline $\begin{array}{l}\text { Blumea } \\
\text { balsamifera (Linn.)DC. }\end{array}$ & 20 & $\begin{array}{c}1.198 \pm 0.023 * \\
* *\end{array}$ & 25.96 & $2.751 \pm 0.049$ & NA \\
\hline $\begin{array}{l}\text { Ciausena } \\
\text { lansium(Lour.)Skeels(lea } \\
\text { ves) }\end{array}$ & 10 & $\begin{array}{c}1.368 \pm 0.037 * \\
*\end{array}$ & 15.45 & $2.892 \pm 0.192$ & \\
\hline DMSO & 0 & $1.618 \pm 0.142$ & & $2.771 \pm 0.123$ & \\
\hline Atropa belladonna $\mathrm{L}$. & 160 & $1.593 \pm 0.046$ & 1.50 & $2.622 \pm 0.068$ & 4.35 \\
\hline $\begin{array}{l}\text { Tylophora } \\
\text { ovata(Lindl.)Hook.ex } \\
\text { Stend. }\end{array}$ & 160 & $1.613 \pm 0.156$ & 0.29 & $2.577 \pm 0.220$ & 6.01 \\
\hline DMSO & 0 & $1.618 \pm 0.142$ & & $2.838 \pm 0.220$ & \\
\hline 3TC(Posive control) & 80 & $\begin{array}{c}1.548 \pm 0.055^{*} \\
* *\end{array}$ & 30.16 & $\begin{array}{c}1.234 \pm 0.04 * \\
* *\end{array}$ & 43.31 \\
\hline DMSO & 0 & $2.217 \pm 0.122$ & & $2.174 \pm 0.10$ & \\
\hline
\end{tabular}

Note: NA, Inhibitory effects of 20 species of herbs on $\mathrm{HBsAg}$ and $\mathrm{HBeAg}$ secretion not tested. NT, toxic to cells and not tested. ND, the highest non-toxic concentraion can not be determined. $* * * \mathrm{P}<0.001 ; * * \mathrm{P}<0.01, * \mathrm{P}<0.05$, as compared with the control group (DMSO).

\subsection{The inhibitory effects of Ethanol extract of $\mathrm{H}$. japonica on HBsAg and $\mathrm{HBeAg}$}

In addition, the inhibitory effects of H.japonica on $\mathrm{HBeAg}$ and HBsAg had better results compared with 3TC and Kushenin.. The results indicated that the ethanol extract of $H$. japonica did not have any inhibition on HBsAg; whereas, the ethanol extract of $H$. japonica had obvious inhibitory effects on $\mathrm{HBeAg}$ secretion, and the highest non-toxic concentration of inhibition rate was $69.06 \%$, which was higher than that of Kushenin. The IC50 value was $14.27 \mu \mathrm{g} / \mathrm{mL}$ and the therapeutic index (TI) $>2$ (table 6); therefore, the ethanol extract of $H$. japonica had the further research value.

Table 2. The inhibitory effects of H. japonica, 3-TC and Kushenin on HBsAg and HBeAg Secretion in HepG2 2.2.15

\begin{tabular}{|c|c|c|c|c|c|c|}
\hline \multirow{2}{*}{ Drugs } & \multirow{2}{*}{$\begin{array}{c}\text { Concentration } \\
(\mathrm{ug} / \mathrm{mL})\end{array}$} & \multicolumn{2}{|c|}{ HBeAg } & \multicolumn{2}{|c|}{ HBsAg } & \multirow{2}{*}{$\begin{array}{c}\text { Cell viability } \\
(\%)\end{array}$} \\
\hline & & OD450 & $\begin{array}{c}\text { Inhibition } \\
\text { rate } \\
(\%)\end{array}$ & OD450 & $\begin{array}{l}\text { Inhibition } \\
\text { rate } \\
(\%)\end{array}$ & \\
\hline & 80 & --- & ---- & --- & --- & $51.33 \pm 0.113$ \\
\hline & 40 & $0.538 \pm 0.018^{* *}$ & 69.06 & $2.443 \pm 0.197$ & 1.17 & $104.70 \pm 0.253$ \\
\hline \multicolumn{7}{|l|}{ Ethanol } \\
\hline & $\begin{array}{l}20 \\
10\end{array}$ & $\begin{array}{l}0.847 \pm 0.123^{* *} \\
0.917 \pm 0.004^{* *}\end{array}$ & $\begin{array}{l}51.64 \\
47.28\end{array}$ & $\begin{array}{l}1.937 \pm 0.211 \\
1.941 \pm 0.420\end{array}$ & $\begin{array}{l}0.73 \\
0.52\end{array}$ & $\begin{array}{l}111.91 \pm 0.189 \\
97 . .35 \pm 0.218\end{array}$ \\
\hline \multirow{5}{*}{$\begin{array}{l}\text { H. japon } \\
\text { ica }\end{array}$} & 5 & $1.166 \pm 0.241^{* *}$ & 32.92 & $1.944 \pm 0.006$ & 0.34 & $101.20 \pm 0.412$ \\
\hline & 2.5 & $1.421 \pm 0.081 * *$ & 18.27 & $1.954 \pm 0.014$ & -0.13 & $95.05 \pm 0.319$ \\
\hline & 1.25 & $1.532 \pm 0.190^{* *}$ & 11.89 & $1.959 \pm 0.332$ & -0.43 & $98.07 \pm 0.362$ \\
\hline & 0 & $1.739 \pm 0.109$ & 0 & $1.951 \pm 0.042$ & 0 & 100 \\
\hline & 80 & $1.330 \pm 0.060$ & 0.58 & $1.234 \pm 0.040^{* *}$ & 43.33 & $103.47 \pm 0.114$ \\
\hline \multicolumn{7}{|l|}{ 3-TC } \\
\hline & 40 & $1.278 \pm 0.050$ & 4.47 & $1.320 \pm 0.140 * *$ & 39.30 & $106.43 \pm 0.067$ \\
\hline & 20 & $1.375 \pm 0.140$ & -2.82 & $1.638 \pm 0.090 * *$ & 24.65 & $105.18 \pm 0.312$ \\
\hline & 0 & $1.338 \pm 0.090$ & 0 & $2.174 \pm 0.100$ & 0 & 100 \\
\hline \multirow{4}{*}{$\begin{array}{l}\text { Kushen } \\
\text { in }\end{array}$} & 3000 & $1.352 \pm 0.090^{* *}$ & 37.40 & $0.883 \pm 0.110^{* *}$ & 64.99 & $108.3 \pm 0.215$ \\
\hline & 1500 & $1.748 \pm 0.110^{* *}$ & 19.06 & $1.321 \pm 0.330^{* *}$ & 47.66 & $96.7 \pm 0.163$ \\
\hline & 7500 & $1.878 \pm 0.160^{* *}$ & 13.04 & $1.888 \pm 0.180^{* *}$ & 25.17 & $101.4 \pm 0.068$ \\
\hline & 0 & $2.159 \pm 0.070$ & 0 & $2.523 \pm 0.070$ & 0 & 100 \\
\hline
\end{tabular}

Note: HepG2 2.2.15 cells were cultured in the presence of Hypericum japonica,3-TC and Kusheninat at various concentrations for 7 days. The $\mathrm{HBsAg}$ and $\mathrm{HBeAg}$ in the supernatants were quantified using specific ELISA kits. Data are presented as mean \pm S.D. of three experiments. ${ }^{* *} \mathrm{P}<0.01$, ${ }^{*} \mathrm{P}<$ 0.05 , as compared with the no drug control group.

3.3 Inhibitory effects of different fractions from $\mathrm{H}$. japonica on $\mathrm{HBeAg}$ 
The H.japonica ethanol extracts were acquired by trichloromethane, ethyl acetate, n-butanol, and water, respectively, to obtain their different fractions. The four fractions from H.japonica ethanol extracts were tested for anti-HBV activity by MTT and ELISA methods, respectively. Results indicated it had the highest inhibition rate of ethyl acetate fraction on the secretion of HBeAg in HepG2 2.2.15 cell line(table 3).This fraction showed $63.46 \%$ of inhibition rate, $111.07 \mu \mathrm{g} / \mathrm{mL}$ of IC50 value and $\mathrm{TI}>2$ (table 6 ). Consequently, it is necessary to isolate further the ethyl acetate fraction of $H$. japonica, which has the highest inhibition rate on the secretion of HBeAg in HepG2 2.2.15 cell line.

Table 3. The inhibitory effects of different fractions from $H$. japonica on HBeAg Secretion in HepG2 2.2.15 cells ( $\mathrm{n}=$

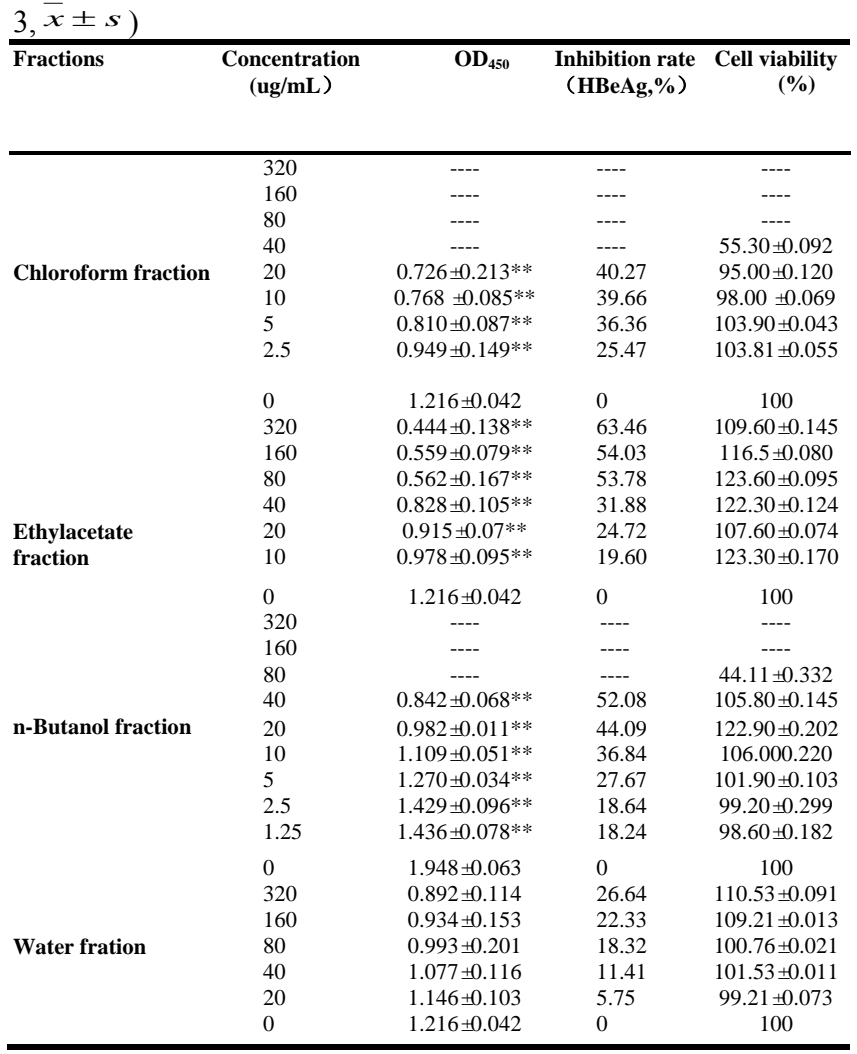

Note: ----, toxic to cells and not tested, $* * \mathrm{P}<0.01,{ }^{*} \mathrm{P}<0.05$, as compared with the no drug control group.

\subsection{The inhibitory effects of different parts of ethyl acetate fraction on $\mathrm{HBeAg}$}

The next step was to isolate the ethyl acetate fraction, as well as obtain 3 parts (YZ1,YZ2 and YZ3) with the active anti-hepatitis $B$ virus by the way of silica gel chromatography. Looking at Table 4, we see that, YZ2 is of the highest inhibition rate on the secretion of $\mathrm{HBeAg}$ in HepG2 2.2.15 cell line. This part indicated that it had $71.57 \% \%$ of inhibition rate, $85.21 \mu \mathrm{g} / \mathrm{mL}$ of IC50 value and $\mathrm{TI}>2$ (table 6 ).

Table 4. The different inhibition parts of ethyl acetate fraction from H. japonicaon on HBeAg secretion in HepG2 2.2.15

$\operatorname{cells}(\mathrm{n}=3, \bar{x} \pm s)$

\begin{tabular}{ccccc}
\hline $\begin{array}{c}\text { Different parts of } \\
\text { ethyl acetate fraction }\end{array}$ & $\begin{array}{c}\text { Concentration } \\
(\boldsymbol{\mu} / \mathrm{mL})\end{array}$ & $\mathbf{O D}_{450}$ & $\begin{array}{c}\text { Inhibition rate } \\
(\mathbf{H B e A g}, \%)\end{array}$ & $\begin{array}{c}\text { Cell viability } \\
(\%)\end{array}$ \\
\hline & 160 & ---- & --- & $60.40 \pm 0.151$ \\
\hline
\end{tabular}

\begin{tabular}{ccccc}
\hline & 80 & $0.776 \pm 0.028^{* *}$ & 44.90 & $103.30 \pm 0.208$ \\
$\mathbf{Y Z}_{1}$ & 40 & $1.029 \pm 0.015^{* *}$ & 26.90 & $105.50 \pm 0.012$ \\
& 20 & $1.031 \pm 0.039^{* *}$ & 26.70 & $98.40 \pm 0.398$ \\
10 & $1.134 \pm 0.065^{* *}$ & 19.40 & $121.10 \pm 0.359$ \\
& 0 & $1.407 \pm 0.009$ & 0 & 100 \\
& 160 & $0.380 \pm 0.080^{* *}$ & 71.57 & $95.60 \pm 0.148$ \\
& 80 & $0.818 \pm 0.017^{* *}$ & 38.85 & $104.80 \pm 0.262$ \\
& 40 & $0.907 \pm 0.045^{* *}$ & 32.20 & $108.90 \pm 0.319$ \\
& 20 & $1.061 \pm 0.058^{* *}$ & 20.66 & $111.50 \pm 0.217$ \\
& 10 & $1.077 \pm 0.069^{* *}$ & 19.51 & $103.10 \pm 0.570$ \\
& 0 & $1.338 \pm 0.085$ & 0 & 100 \\
& 160 & ---- & --- & $40.00 \pm 0.071$ \\
& 80 & ---- & --- & $78.70 \pm 0.197$ \\
& 40 & $0.798 \pm 0.033^{* *}$ & 40.40 & $107.20 \pm 0.117$ \\
& 20 & $0.991 \pm 0.042^{* *}$ & 25.90 & $107.50 \pm 0.352$ \\
& 10 & $1.034 \pm 0.018^{* *}$ & 22.70 & $97.70 \pm 0.059$ \\
& 0 & $1.338 \pm 0.085$ & 0 & 100 \\
\hline
\end{tabular}

Note: ----, toxic to cells and not tested, ${ }^{*} \mathrm{P}<0.01,{ }^{*} \mathrm{P}<0.05$, as compared with the no drug control group.

\subsection{The isolation of the compounds with anti- HBV activities}

Four compounds were separated from the ethyl acetate fraction of $H$. japonica. Their structures were identified as Vincetoxin side B, (2R,3R) double hydrogen quercetin-7-O- $\alpha$-L-rhamnoside, quercitrin, and 1,3,6,7tetrahydroxy-9H-xanthen-9-one(figure 1). Among the 4 compounds, 1, 3, 6, 7-tetrahydroxy-9H-xanthen-9-one exhibited the highest activity in inhibiting secretion of HBeAg in HepG2 2.2.15 cells (table 5). The results indicated a $67.66 \%$ of inhibition rate, $40.25 \mu \mathrm{g} / \mathrm{mL}$ of IC50 value and TI $>2$ (table 6).

Table 5. The inhibitory effect of the four compounds of the ethyl acetate fraction from $H$. japonica on $\mathrm{HBeAg}$ secretion in

\begin{tabular}{|c|c|c|c|c|}
\hline $\begin{array}{l}\text { Different parts of } \\
\text { ethyl acetate fraction }\end{array}$ & $\begin{array}{c}\text { Concentration } \\
(\mu \mathrm{g} / \mathrm{mL})\end{array}$ & $\mathrm{OD}_{50}$ & $\begin{array}{r}\text { Inhibition rate } \\
(\mathrm{HBeAg}, \%)\end{array}$ & $\begin{array}{c}\text { Cell viability } \\
(\%)\end{array}$ \\
\hline \multirow{7}{*}{ Compound 1} & 40 & $\overline{----}$ & --- & $28.10 \pm 0.131$ \\
\hline & 20 & --- & --- & $35.80 \pm 0.151$ \\
\hline & 10 & $1.103 \pm 0.055$ & -0.094 & $100.10 \pm 0.145$ \\
\hline & 5 & $1.055 \pm 0.063$ & -0.046 & $109.30 \pm 0.020$ \\
\hline & 2.5 & $1.027 \pm 0.046$ & -0.018 & $101.90 \pm 0.395$ \\
\hline & 0 & $1.009 \pm 0.034$ & 0 & 100 \\
\hline & 40 & $0.798 \pm 0.042 * *$ & 22.60 & $119.40 \pm 0.076$ \\
\hline \multirow{5}{*}{ Compound 2} & 20 & $0.857 \pm 0.070 * *$ & 17.00 & $100.40 \pm 0.111$ \\
\hline & 10 & $0.851 \pm 0.025 * *$ & 17.50 & $114.90 \pm 0.242$ \\
\hline & 5 & $0.852 \pm 0.065^{* *}$ & 17.40 & $122.70 \pm 0.019$ \\
\hline & 2.5 & $0.971 \pm 0.074 * *$ & 5.80 & $108.60 \pm 0.542$ \\
\hline & 0 & $1.032 \pm 0.182$ & 0 & 100 \\
\hline \multirow{7}{*}{ Compound 3} & 80 & $0.803 \pm 0.249 * *$ & 22.10 & $95.80 \pm 0.086$ \\
\hline & 40 & $0.900 \pm 0.243^{* *}$ & 12.80 & $103.40 \pm 0.137$ \\
\hline & 20 & $0.974 \pm 0.257 * *$ & 5.60 & $101.50 \pm 0.035$ \\
\hline & 10 & $1.032 \pm 0.132$ & -0.012 & $105.60 \pm 0.149$ \\
\hline & 5 & $1.035 \pm 0.223$ & -0.321 & $105.50 \pm 0.157$ \\
\hline & 2.5 & $1.033 \pm 0.246$ & -0.145 & $111.80 \pm 0.140$ \\
\hline & 0 & $1.032 \pm 0.182$ & 0 & 100 \\
\hline \multirow{7}{*}{ Compound 4} & 80 & $0.334 \pm 0.057 * *$ & 67.66 & $101.80 \pm 0.577$ \\
\hline & 40 & $0.571 \pm 0.101 * *$ & 44.65 & $117.30 \pm 0.081$ \\
\hline & 20 & $0.682 \pm 0.171^{* *}$ & 33.81 & $103.10 \pm 0.234$ \\
\hline & 10 & $0.853 \pm 0.098^{* *}$ & 17.31 & $112.50 \pm 0.341$ \\
\hline & 5 & $0.960 \pm 0.161^{* *}$ & 6.94 & $105.40 \pm 0.264$ \\
\hline & 2.5 & $1.019 \pm 0.042 * *$ & 1.23 & $99.10 \pm 0.077$ \\
\hline & 0 & $1.032 \pm 0.182$ & 0 & 100 \\
\hline
\end{tabular}

Note: ----, toxic to cells and not tested, ${ }^{* * \mathrm{P}<0.01},{ }^{*} \mathrm{P}<0.05$, as compared with the no drug control group. 
Table 6. Evaluation of anti-HBV efficacy of the active site and isolated compound of $\mathrm{H}$. japonica

\begin{tabular}{cccc}
\hline Extract & $\mathrm{IC}_{50}(\mu \mathrm{g} / \mathrm{mL})$ & $\mathrm{TC}_{50}(\mu \mathrm{g} / \mathrm{mL})$ & $\mathrm{TI}$ \\
\hline $\begin{array}{c}\text { H. japonica } \text { ethanol } \\
\text { extract }\end{array}$ & 14.27 & $>40$ & $>2$ \\
Ethylacetate extract & 111.07 & $>320$ & $>2$ \\
$\mathbf{Y Z}_{2}$ & 85.21 & $>180$ & $>2$ \\
Compound 4 & 40.25 & $>100$ & $>2$
\end{tabular}

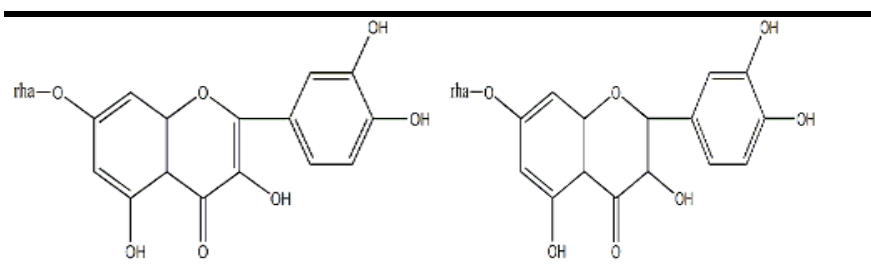

Compound 1(vincetoxicoside B) Compound 2((2R,3R)-taxifolin-7-O- a -Lrhamnoside)

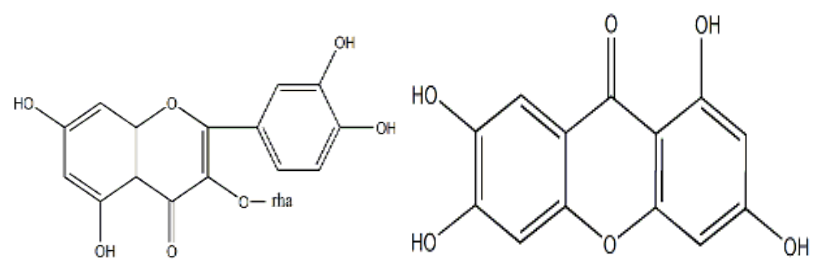

Compound 3 (quercitrin) Compound 4 (1,3,6,7-tetrahydroxy-9H-xanthen-9one)

Fig.1. Chemical structures of the compounds.

vincetoxicoside $B$

mp $174 \sim 180{ }^{\circ} \mathrm{C}$, ESI-MS m/s:447[M-H]-, $1 \mathrm{H}-$ $\mathrm{NMR}(400 \mathrm{MHz}, \mathrm{DMSO}-\mathrm{d} 6) \quad \delta: 1.14(3 \mathrm{H}, \mathrm{d}, \mathrm{J}=6.0 \mathrm{~Hz}$, rha$\mathrm{Me}), \quad 5.55(1 \mathrm{H}, \mathrm{J}=1.7 \mathrm{~Hz}, \quad$ rhaH-1 $), \quad 6.41(1 \mathrm{H}, \mathrm{m}, \mathrm{H}-6)$, $6.79(1 \mathrm{H}, \mathrm{d}, \mathrm{J}=2.1 \mathrm{~Hz}, \quad \mathrm{H}-8), 6.90(1 \mathrm{H}, \mathrm{d}, \mathrm{J}=8.1 \mathrm{~Hz}, \quad \mathrm{H}-5)$, $7.57\left(1 \mathrm{H}, \mathrm{dd}, \mathrm{J}=8.6 \mathrm{~Hz}, \mathrm{H}-6^{\prime}\right), 7.72\left(1 \mathrm{H}, \mathrm{d}, \mathrm{J}=2.1 \mathrm{~Hz}, \mathrm{H}-2^{\prime}\right)$. 13C-NMR(100MHz, DMSO-d6) $\delta: 18.0\left(\mathrm{C}-6^{\prime \prime}\right), 69.9\left(\mathrm{C}-5^{\prime \prime}\right)$, 70.0(C-2"), 70.2(C-3"), 71.6(C-4"), 94.2(C-8), 98.4(C-6), 98.8(C-1"), 104.6(C-10), 115.2(C-6'), 115.6(C-5'), 120.1(C-2'), 121.8(C-1'), 136.1(C-3), 145.1(C$\left.3^{\prime}\right), 147.5\left(\mathrm{C}-4^{\prime}\right), \quad$ 147.9(C-2), 155.7(C-9), 160.4(C-7), 161.4(C-5), 176.0(C-4).

(2R,3R) -taxifolin-7-O- $\alpha$-L-rhamnoside

mp $198 \sim 200^{\circ} \mathrm{C} .1 \mathrm{H}-\mathrm{NMR}(400 \mathrm{MHz}, \mathrm{DMSO}-\mathrm{d} 6) \delta: 6.76$ $\left(1 \mathrm{H}, \mathrm{s}, \mathrm{J}=11.60 \mathrm{~Hz}, \mathrm{H}-2^{\prime}\right), 6.75\left(1 \mathrm{H}, \mathrm{d}, \mathrm{J}=8.1 \mathrm{~Hz}, \mathrm{H}-5^{\prime}\right)$, $6.75\left(1 \mathrm{H}, \mathrm{dd}, \mathrm{J}=1.0,8.0 \mathrm{~Hz}, \mathrm{H}-6^{\prime}\right), 6.17(1 \mathrm{H}, \mathrm{d}, \mathrm{J}=1.9 \mathrm{~Hz}, \mathrm{H}-$ 8), $6.12(1 \mathrm{H}, \mathrm{d}, \mathrm{J}=1.8 \mathrm{~Hz}, \mathrm{H}-6), 5.45(1 \mathrm{H}, \mathrm{d}, \mathrm{J}=10.3 \mathrm{~Hz}, \mathrm{H}-2)$, $3.92(1 \mathrm{H}, \mathrm{d}, \mathrm{J}=11.6 \mathrm{~Hz}, \mathrm{H}-3), 3.38(1 \mathrm{H}, \mathrm{dd}, \mathrm{J}=1.7,3.4 \mathrm{~Hz}$, RhaH-2),3.36(1H, dd, J=3.1,9.4 Hz, RhaH3),3.28(1H,m,RhaH-5),3.26(1H,t,J=10.0 Hz, RhaH4), $1.10(3 \mathrm{H}, \mathrm{d}, \mathrm{J}=6.0 \mathrm{~Hz}$, Rha-Me); 13C-NMR(DMSO-d6, $100 \mathrm{MHz}) \delta: 17.9(\mathrm{C}-6 "), 68.9\left(\mathrm{C}-5^{\prime \prime}\right), 69.8\left(\mathrm{C}-2^{\prime \prime}\right), 70.2(\mathrm{C}-$ $\left.3^{\prime \prime}\right), 71.5\left(\mathrm{C}-4^{\prime \prime}\right), 75.5(\mathrm{C}-3), 83.1(\mathrm{C}-2), 95.4(\mathrm{C}-8), 96.5(\mathrm{C}-$ 6), 98.2(C-1" $), 102.0(\mathrm{C}-10), \quad 115.1\left(\mathrm{C}-5^{\prime}\right), \quad 115.4\left(\mathrm{C}-2^{\prime}\right)$, 119.4(C-6' $\quad 127.8\left(\mathrm{C}-1^{\prime}\right), 145.0\left(\mathrm{C}-3^{\prime}\right), \quad 145.8(\mathrm{C}-$ $\left.4^{\prime}\right), 162.3(\mathrm{C}-9), 162.8(\mathrm{C}-5), 164.1(\mathrm{C}-7), 198.6(\mathrm{C}-4)$.

Quercitrin

$\mathrm{mp} 180 \sim 181^{\circ} \mathrm{C} . \mathrm{ESI}-\mathrm{MS} \mathrm{m} / \mathrm{z}: 447[\mathrm{M}-\mathrm{H}]-$. 1H-NMR (400 MHz, DMSO-d6) $\delta: 7.30(1 \mathrm{Hd}, \quad \mathrm{J}=2.0 \mathrm{HzH}-$ $\left.2^{\prime}\right) 7.29\left(1 \mathrm{HddJ}=8.42 .0 \mathrm{HzH}-6^{\prime}\right) 6.86(1 \mathrm{H}, \mathrm{d}, \mathrm{J}=8.4 \mathrm{~Hz}, \mathrm{H}-$ $\left.5^{\prime}\right), 6.39(1 \mathrm{H}, \mathrm{d}, \mathrm{J}=2.0 \mathrm{~Hz}, \mathrm{H}-8), 6.20(1 \mathrm{H}, \mathrm{d}, \mathrm{J}=2.0 \mathrm{~Hz}, \mathrm{H}-6)$, 5.24(1H, d, J=1.6Hz, H-l"), 3.97(1H, brsH-2"), 3.51(1H, $\left.\mathrm{dd}, \quad \mathrm{J}=9.53 .2 \mathrm{HzH}-3^{\prime \prime}\right), \quad 3.15\left(1 \mathrm{H}, \quad \mathrm{t}, \quad \mathrm{J}=10 \mathrm{~Hz}, \mathrm{H}-4^{\prime \prime}\right)$, $3.22\left(1 \mathrm{H}, \mathrm{dp}, \mathrm{J}=9.56 .1 \mathrm{HzH}-5^{\prime \prime}\right), 0.88(3 \mathrm{H}, \mathrm{d}, \mathrm{J}=6.1 \mathrm{~Hz}, \mathrm{H}-$ 6" ); 13C-NMR (DMSO-d6, 100MHz) $\delta: 17.7$ (C-6"), $70.0\left(\mathrm{C}-5^{\prime \prime}\right), 70.3\left(\mathrm{C}-2^{\prime \prime}\right), 70.6\left(\mathrm{C}-3^{\prime \prime}\right), 71.5\left(\mathrm{C}-4^{\prime \prime}\right), 101.1(\mathrm{C}-$ $\left.1^{\prime \prime}\right), \quad 120\left(\mathrm{C}-6^{\prime}\right), \quad 115.5\left(\mathrm{C}-5^{\prime}\right), \quad 148.4\left(\mathrm{C}-4^{\prime}\right), 145.2\left(\mathrm{C}-3^{\prime}\right)$, 115.6(C-2'), $11.1\left(\mathrm{C}-1^{\prime}\right), \quad 104.1(\mathrm{C}-10), \quad 157.3(\mathrm{C}-9)$, 93.6(C-8), 164.2(C-7), 98.7(C-6),161.3(C-5), 177.8(C-4), 134.2(C-3), 156.4(C-2).

1,3,6,7-tetrahydroxy-9H-xanthen-9-one

$\mathrm{mp}>300{ }^{\circ} \mathrm{C}$, ESI-MS m/z: 260[M-H]-. 1H-NMR (400MHz, DMSO-d6) $\delta: 7.36(1 \mathrm{H}, \quad \mathrm{d}, \quad \mathrm{J}=8 \mathrm{~Hz}, \mathrm{H}-7)$, $6.54(1 \mathrm{H}, \mathrm{d}, \mathrm{J}=8 \mathrm{~Hz}, \mathrm{H}-5), 6.32(1 \mathrm{H}, \mathrm{s}, \mathrm{H}-4), 6.14(1 \mathrm{H}, \mathrm{s}, \mathrm{H}-$ 2); 13C-NMR(DMSO-d6, 100MHz) $\delta: 93.6(\mathrm{C}-4), 97.6(\mathrm{C}-$ 2), 101.5(C-9a), 102.5(C-5), 107.8(C-8), 111.5(C-8a), 143.9(C-7), 151.2(C-4b), 154.7(C-6), 157.3(C-4a), 162.5(C-1), 164.6(C-3), 178.8(C-9).

\subsection{The inhibitionry effects on serum DHBV-DNA of compound 4}

we conducted a qualitatively evaluated research using duck hepatitis B virus (DHBV) model in vivo [12-13]. The results showed that the compound 4 has no direct inhibitory effect on HBV-DNA in duck experiment. This indicated that compound 4 had the effect of anti-HBV activity through inhibiting the secretion of $\mathrm{HBeAg}$.

\section{Discussion}

In this study, we conducted a qualitatively evaluated research using DHBV model in vivo, Ethanol extracts from $F$. dibotrys, $P$. saxatile and $H$. japonica, as well as the compound 4. Beijing ducks were infected with duck hepatitis B virus (DHBV) and they were used to evaluate anti-HBV activity. Treatments began on the 7 th-day post infection. The ducks were infected with $26 \mathrm{mg} / \mathrm{kg}$ (high dose groups), $6.5 \mathrm{mg} / \mathrm{kg}$ (low dose groups) of ethanol extracts from $F$. dibotrys, $P$. saxatile, respectively. Drugs were given twice a day for ten consecutive days. The results indicated that the high dose groups, which were treated with ethanol extracts of $P$. saxatile or H. japonica, had anti-HBV effects and almost no sign of disease rebound after drug withdrawing. Treatment with ethanol extract from $F$. dibotrys also had certain levels of inhibitory effect on HBV-DNA, but the disease rebound after the withdrawing of treatment. The compound 4 has no direct inhibitory effect on HBV-DNA in duck experiment.

To our knowledge, this is the first report to show the 1 , 3, 6, 7-tetrahydroxy-9H-xanthen-9-one has anti-HBV activity and low cytotoxicity in the in vitro assay, and our $P$. saxatile has anti-HBV activity in vivo.

\section{References}

1. María L. Cuestas \& Verónica L. Mathet. Drug delivery systems and liver targeting for improved pharmacotherapy of the Hepatitis B virus (HBV) infection. Pharm Res, 27, 1184-1202 (2010)

2. Su-zhen Jiang, Jia-jia Zheng. Hepatitis B Virus S Promoter Deletionin Hepatocellular Carcinoma. Infection Inte national, 1, 14-23 (2012) 
3. Hepatitis B. World Health Organization. World Health Org. 204 (2012)

4. Park NH, Song IH. Chronic hepatitis B in hepatocarcinogenesis.Pos. Med J, 82(970), 507515.(2006)

5. Westland C, Delandy W. Hepatitis B virus genotypes and virologic response in 694 patients in phase III studies of adefovir dipivoxil.Gas, 125(1), 107-116 (2003)

6. Wright TL.Introduction to chronic hepatitis B infection.Am J Gastl, 101, 1-6 (2006)

7. Ceen-Ming Tang, Tung On Yau. Management of chronic hepatitis B infection: Currenttreatment guidelines, challenges, and new developmentsWorld J Gastroenterol, 20(20), 6262-6278 (2014)

8. Han YX, Xue R,Zhao W. Antiviral therapertic efficacy of foscamet inhepatitis B virus infection. Antiv Res, 68(3), 147-153 (2005)

9. Shin MS, Kang EH. A flavonoid from medicinal plants blocks hepatitis Bvirus-e antigen secretion in HBV-infected hepatocytes. Antiv Res, 67(3), 163168 (2005)

10. Zembower DE, Lin YM. Robustaflavone, a potential non-nucleoside anti-hepatitis B agent. Antiv Res, 39(2),81-88 (1998)

11. Zhou Z,Zhang Y,Ding XR. Protocatechuic aldehyde inhibits hepatitis Bvirus replication both in vitro and in vivo. Antiv Res, 74(1), 59-64 (2007)

12. Addison WR, Wong WW. A quantitative competitive PCR assay for thecovalently closed circular form of the duck hepatitis B virus. Antiv Res, 48(1), 27-37 (2000)

13. Le Guerhier F, Thermet A. Antiviral effect of adefovir in combination with aDNA vaccine in the duck hepatitis B virus infection model.J. Hepa, 38(3), 328-334 (2003) 